\title{
The influence of plasticizers on the release of theophylline from microporous-controlled tablets
}

\author{
W.J. Lin ${ }^{\mathrm{a}, *}$, H.K. Lee ${ }^{\mathrm{a}}$, D.M. Wang ${ }^{\mathrm{b}}$

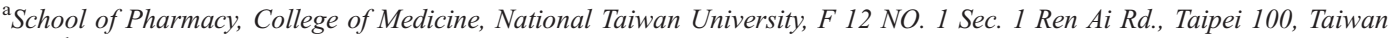 \\ ${ }^{\mathrm{b}}$ Department of Chemical Engineering, College of Engineering, National Taiwan University, Taipei 100, Taiwan
}

Received 5 May 2004; accepted 15 August 2004

Available online 11 September 2004

\begin{abstract}
The aim of present work was to investigate the influence of plasticizer on the release of theophylline from microporouscontrolled tablets. Three plasticizers, acetyltributyl citrate (ATBC), castor oil, and triacetin, were included in this study. These plasticizers reduced the crystallinity of poly( $\varepsilon$-caprolactone) (PCL)/poly(ethylene glycol) (PEG)-blended films, and the most prominent change of enthalpy of fusion was the film plasticized by triacetin. This might be due to triacetin penetrating into both PCL and PEG domains. However, the lipophilic property of castor oil only allowed it to alter the crystallization of hydrophobic PCL domain. The Young's modulus and the tensile strength of films showed a decreased tendency while increasing the amount of plasticizer. The change of elongation of plasticized blended films was irregular and was dependent of the type of plasticizer. The size of micropores formed in the presence of plasticizer was larger than those micropores formed in its absence. The fatty plasticizer, castor oil, altered the thermal and mechanical performance and pore size of films via soluble in PCL domain, which resulted in the release of theophylline from castor oil plasticized-coated tablets, which in turn enhanced and closed to a constant release pattern.
\end{abstract}

(C) 2004 Elsevier B.V. All rights reserved.

Keywords: Microporous-controlled tablets; Poly( $\varepsilon$-caprolactone); Poly(ethylene glycol); Plasticizer

\section{Introduction}

Blended polymers play an important role in developing the microporous-controlled tablet. There can be combined two types of polymers in the blended

* Corresponding author. Tel.: +886 2 23123456x8396; fax: +886223916126 .

E-mail address: wjlin@ha.mc.ntu.edu.tw (W.J. Lin). films: one is remained in the end-use, and another is removed as a pore-forming agent to produce porous structure for drug release [1]. The advantage of blending of pore-forming agent in microporouscontrolled tablets avoids using high-technical laser beam to drill an orifice for drug release; in addition, it is easily fabricated via traditional film coating technique [2]. Therefore, the choosing of proper solvents for polymer dissolving becomes an important issue. It is known that a good solvent can enhance 
polymer chain extension and entanglement in the polymer solution. Felton and McGinity [3] mentioned that both the interaction between polymer chains and solvents influence polymer random coil structure. The stronger the interaction is, the longer the end-to-end distance is. This turns out the films with better mechanical behavior.

Plasticizer has been used to modify not only the mechanical properties but also the thermal property, water absorption behavior, and adhesive property of polymeric films [4]. All of these properties affect the strength of coating films and the integrity of final products, which further affect drug release performance. Many compounds can be acted as a function of plasticizer including poly(ethylene glycol) [5], propylene glycol [6], sorbitol [7], urea [8], oil [9], citrate [10], adipate [11], and phthalate [12], etc. These plasticizers perform different outcomes due to their intrinsic properties and the interaction with polymers. The water-soluble and water-insoluble citrate-type plasticizers show different influence on plasticization time, which results in different release rates of drug $[13,14]$. Castor oil has been used as a hydrophobic plasticizer to enhance film permeability in terms of increasing drug release from coated beads [15]. The similar phenomenon has also been found by using a nonfatty plasticizer, triacetin, where the plasticized films with good mechanical properties allow drug permeation [16].

The microporous-controlled tablet has been demonstrated in our previous study, where poly( $\varepsilon$-caprolactone) (PCL) is the major component of film coating material and poly(ethylene glycol) (PEG) is acted as a leachable pore-forming agent [17]. The performance of coating film is strongly influenced by the molecular weight of pore-forming agent and the solvent used for polymer dissolving. Because hydrophobic PCL and hydrophilic PEG have similar solubility parameters $(\sim 20 \mathrm{MPa})$, this allows them to dissolve in the common solvent in order to obtain homogeneous blended films. PCL is a highly crystallized polymer, and it is not feasible for water penetration and drug release. The release of drug from microporouscontrolled tablets is mainly dominated by the pore size and the interconnected channels on the coating films after PEG leaching out in an aqueous medium. The use of plasticizer to modify the thermal and mechanical performance of polymers has been studied a lot; however, the impacts of plasticizer on the performance of microporous-controlled tablets are not revealed. Therefore, the aim of present work was designed to investigate the influence of plasticizer on the performance of coating films and the release character of plasticized, microporous-controlled tablets. Three plasticizers, acetyltributyl citrate (ATBC), castor oil, and triacetin, were included in this study. Theophylline was used as a model drug. The influences of the type and the level of plasticizer on the thermal and mechanical properties of $\mathrm{PCL}_{60 \%}$ $\mathrm{PEG}_{40 \%}$-blended films were evaluated. In addition, the release of theophylline from plasticized microporous-controlled tablets was investigated, and the release mechanism was proposed. The reason to choose $\mathrm{PCL}_{60 \%} / \mathrm{PEG}_{40 \%}$ as the composition of blends in this study is because incorporation of $40 \%(\mathrm{w} / \mathrm{w})$ of pore-forming agent in the blends created quite prominent micropores on the coating film after PEG leaching out, which turned out the microporouscontrolled tablets with efficient drug release rate [17].

\section{Materials and methods}

\subsection{Materials}

PCL $\left(M_{\mathrm{w}} 65,000\right)$, PEG $\left(M_{\mathrm{w}} 10,000\right)$, acetyltributyl citrate (ATBC), and castor oil were from Aldrich Chemical (St. Wisconsin, Milwaukee, USA). Triacetin was from Acros Chemical (New Jersey, USA). Theophylline was from Sigma (St. Louis, MO, USA).

\subsection{Preparation of polymeric films}

Polymeric films were prepared using a solventcasting method as described by the following: PCL and PEG $(40 \% \mathrm{w} / \mathrm{w})$ without or with plasticizer $(5$, 10 , and $20 \% \mathrm{w} / \mathrm{w}$ ) were codissolved in dichloromethane, cast on the glass molds, and dried at $40{ }^{\circ} \mathrm{C}$ for $12 \mathrm{~h}$. The films were removed from the molds and further dried in a desiccator for 3 days to eliminate the residual solvent. Each dried film was then determined their thermal and mechanical properties.

\subsubsection{Differential scanning calorimetry (DSC)}

The enthalpy of fusion and the glass transition temperature $\left(T_{\mathrm{g}}\right)$ of polymeric films were measured 
using a DSC (LT-Modulate DSC 2920, DuPont Instrument, USA). Each sample $(\sim 5 \mathrm{mg})$ was heated to $80{ }^{\circ} \mathrm{C}$ at a rate of $10{ }^{\circ} \mathrm{C} / \mathrm{min}$ (first run), and the enthalpy of fusion was determined from the DSC endotherm. For measurement of the $T_{\mathrm{g}}$, the sample was rapidly quenched, and reheated from -120 to $80{ }^{\circ} \mathrm{C}$ at a heating rate of $10{ }^{\circ} \mathrm{C} / \mathrm{min}$ (second run). The $T_{\mathrm{g}}$ was taken at the midpoint as the heat capacity changed.

\subsubsection{Mechanical testing}

The polymeric films were cut into $6 \times 2 \mathrm{~cm}^{2}$ and clamped on the testing machine (LLOYD-LRX, England). The effective length of the film under stress was kept constant at $3.5 \mathrm{~cm}$, and a speed of $0.5 \mathrm{~mm} / \mathrm{min}$ was used for the measurement. The stress-strain profile was recorded, and three measurements were conducted for each specimen. The tensile strength, percentage elongation, and Young's modulus were computed from the stress-strain profiles.

\subsection{Morphology of microporous films}

The PCL/PEG-blended films with or without castor oil were immersed in deionized water for 14 days, and the medium was replaced every day to assure PEG leaching out. The obtained microporous films were dried in vacuum. The vertical crosssection of microporous films was prepared with a blade under liquid nitrogen, coated under an argon atmosphere with gold/palladium, and observed with a scanning electron microscope (SEM; JEOL JSM6300, Japan).

\subsection{Preparation of coated tablets}

Theophylline ( $150 \mathrm{mg})$ was weighed in an 8-mm diameter of die and compressed directly by IR compressor under $2400 \mathrm{lb}$. force for $10 \mathrm{~s}$. PCL and PEG without or with castor oil $(5,10$, and $20 \% \mathrm{w} / \mathrm{w})$ were weighed and dissolved in dichloromethane. The polymer solution was then coated on the surface of theophylline tablets via a dip-coating method, and the coated tablets were dried in an oven under vacuum for $48 \mathrm{~h}$. The weight of the coating film for each tablet was controlled around $3.5 \% \mathrm{w} / \mathrm{w}$ with respect to the weight of core tablet.

\subsection{In vitro release study}

The release of theophylline from coated tablets was conducted according to the USP XXV basket method. Deionized water was used as the dissolution medium and maintained at $37 \pm 0.5^{\circ} \mathrm{C}$. The stirring speed was set at $100 \mathrm{rpm}$. Samples $(1 \mathrm{ml})$ were withdrawn at specific time points, and the same volume of fresh dissolution medium was replaced. The concentration of theophylline in each sample was determined by validated UV spectrophotometer (Hitachi U-2000, Japan) at $272 \mathrm{~nm}$. In all cases, three runs were carried out for each formulation. The accumulated amount of drug released at each sampling point was corrected with the volume of the dissolution medium. The $70 \%$ of drug released from coated tablets was further fitted by Eq. (1) in order to propose the possible release mechanism [18]

$M_{t} / M_{\infty}=k t^{n}$

where $M_{t}$ : the amount of drug released at time $t ; M_{\infty}$ : total amount of drug in each tablet; $k$ : the release rate constant; $n$ : exponent constant.

The constant $n$ was usually used to relate the release mechanism of drug from dosage forms.

\section{Results and discussion}

\subsection{Properties of plasticized $P C L_{60 \%} / P E G_{40 \%}$ blended films}

\subsubsection{Thermal property}

Fig. 1 shows the glass transition temperature of $\mathrm{PCL}_{60 \%} / \mathrm{PEG}_{40 \%}$-blended films plasticized by different types and levels of plasticizers. The $T_{\mathrm{g}}$ of unplasticized $\mathrm{PCL}_{60} \% / \mathrm{PEG}_{40 \%}$ film was measured to be $-60.3{ }^{\circ} \mathrm{C}$. The reduction of $T_{\mathrm{g}}$ by castor oil was not so prominent as by ATBC or triacetin. Fig. 2 shows the enthalpies of fusion of these films. The corresponding value of unplasticized $\mathrm{PCL}_{60} / \mathrm{PEG}_{40 \%}$ film was measured to be $118.9 \mathrm{~J} / \mathrm{g}$. Incorporation of $20 \%(\mathrm{w} / \mathrm{w})$ of each plasticizer, ATBC, castor oil, and triacetin, decreased their enthalpies to 101.0, 95.4, and $75.4 \mathrm{~J} / \mathrm{g}$, respectively. These results indicated that these plasticizers reduced the crystallinity of blended films in terms of reduction of their enthalpies. The most prominent change of enthalpy of fusion was the 


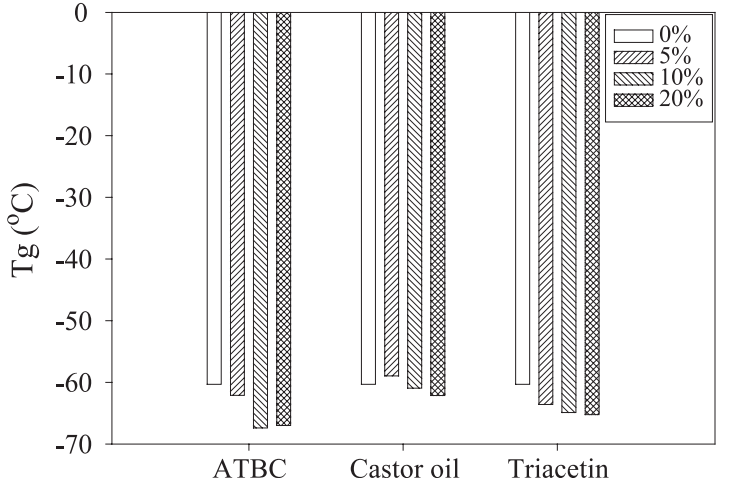

Fig. 1. The glass transition temperature of plasticized $\mathrm{PCL}_{60 \%} /$ $\mathrm{PEG}_{40 \%}$ films.

film plasticized by triacetin. PCL and PEG were highly crystallized polymers with enthalpies of fusion 93.7 and $212.0 \mathrm{~J} / \mathrm{g}$, respectively. It seemed that triacetin penetrated into both PCL and PEG domains to disturb their crystallization. However, the lipophilic character of castor oil only allowed it to alter the crystallization of hydrophobic PCL phase, which resulted in the reduction of enthalpy of fusion not so prominent as triacetin.

\subsubsection{Mechanical evaluation}

Fig. 3 shows the Young's modulus, tensile strength, and elongation of plasticized PCL/PEG films. The Young's modulus of films decreased as the amount of plasticizer increased, and the decrease magnitude was in the order of $\mathrm{ATBC}>$ castor oil $>$ triacetin. The tensile strength of these films showed a decreased tendency as the amount of ATBC and triacetin increased. However, the corresponding values of films plasticized by castor

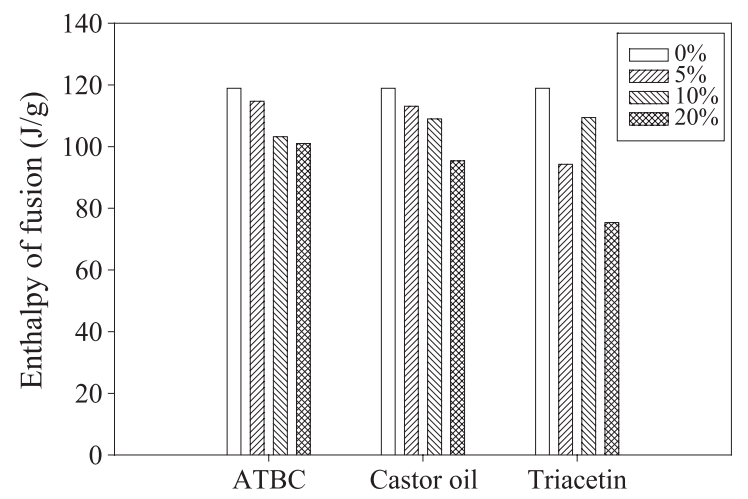

Fig. 2. The enthalpy of fusion of plasticized $\mathrm{PCL}_{60} \% / \mathrm{PEG}_{40 \%}$ films. (a)

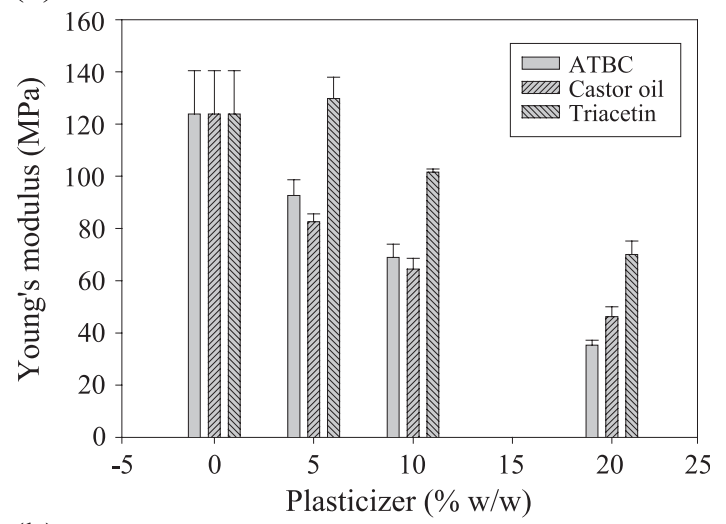

(b)

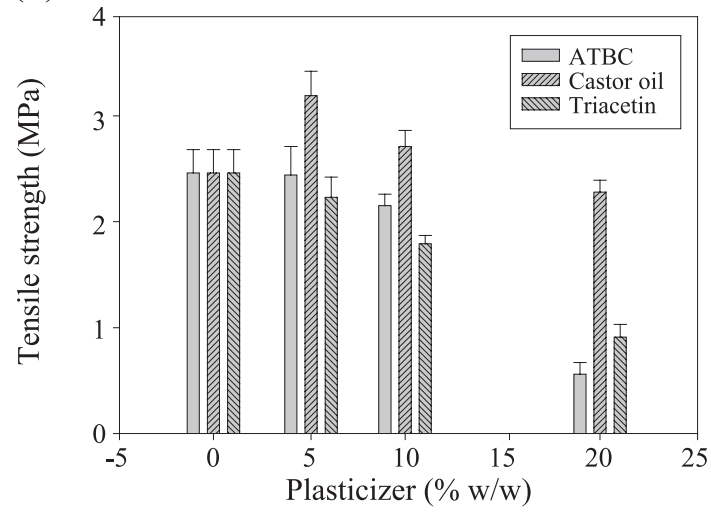

(c)

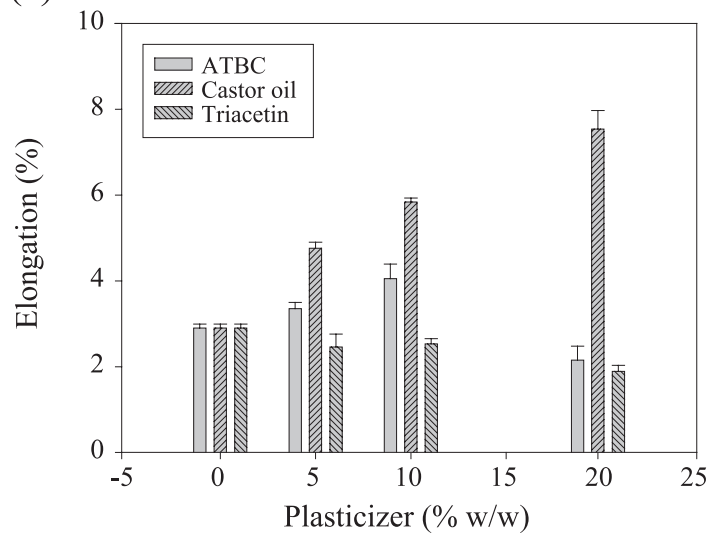

Fig. 3. The mechanical properties of plasticized $\mathrm{PCL}_{60} / \mathrm{PEG}_{40 \%}$ films: (a) Young's modulus, (b) tensile strength, and (c) elongation.

oil were greater or slightly lower than that of unplasticized blended film. The change of elongation of plasticized blended films was irregular and dependent of the type of plasticizer. A slight reduction of elongation was observed from films plasticized by 
triacetin, in reverse, the elongation of castor oilplasticized film showed a significant increased tendency as increasing the amount of plasticizer. This result implied that addition of castor oil could enhance film flexibility via acted as a lubricant among polymer chains. The elongation of films plasticized by ATBC showed an increase followed by a decrease tendency, and a more flexible character was present when $5-10 \%$ of ATBC was added. The increase in elongation at level of $5-10 \%$ ATBC was similar to the result of castor oil, and the decrease of elongation when $20 \%$ ATBC plasticized was the same as the performance of triacetin. This outcome suggested that at low level of ATBC (e.g., 5-10\% w/w), the effect on PCL domain is much prominent as castor oil; however, the effect on PEG domain became enhanced when high level of ATBC (e.g., 20\% w/w) was plasticized. Because PCL was the main component of microporous films after PEG leaching out and because castor oil mainly affected PCL phase according to the thermal data, therefore, castor oil was chosen as the target plasticizer used in the microporous-controlled tablets for the following study.

\subsection{Morphology of microporous films}

Fig. 4 shows the SEM micrographs of microporous films formed by $\mathrm{PCL}_{60 \%} / \mathrm{PEG}_{40 \%}$ blends and plasticized by various level of castor oil $(0,5,10$, and $20 \%$ $\mathrm{w} / \mathrm{w})$ following by leaching in an aqueous medium. Quite difference in micropore size was observed in plasticized-leaching microporous films, where the size of micropores formed in the presence of plasticizer was larger than the size of the micropores formed in its absence, especially when the level of castor oil was higher than $10 \%$. According to the previous study [17], the thermal properties (e.g., Tg, Tm, and the heat of fusion) of PCL/PEG-blended films were almost invariant independent of the amount of PEG blended, and there was no appreciable shift of the IR peak at around $1725 \mathrm{~cm}^{-1}$ corresponding to the carbonylstretching band of PCL. Both results indicated no (a)

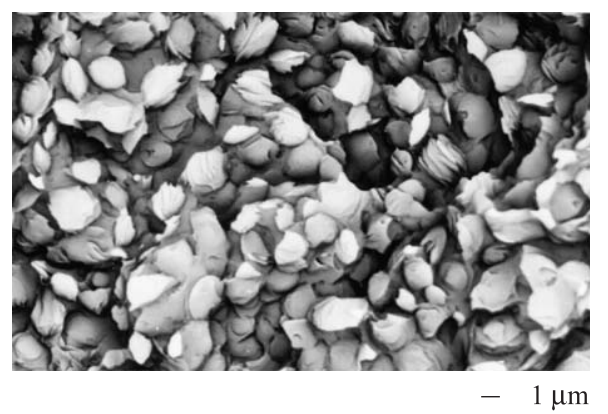

(c)

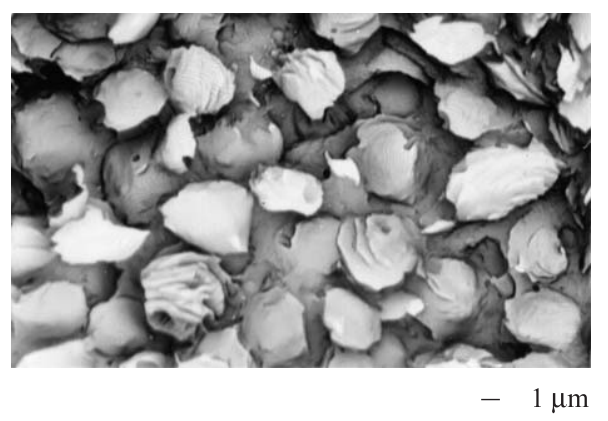

(b)

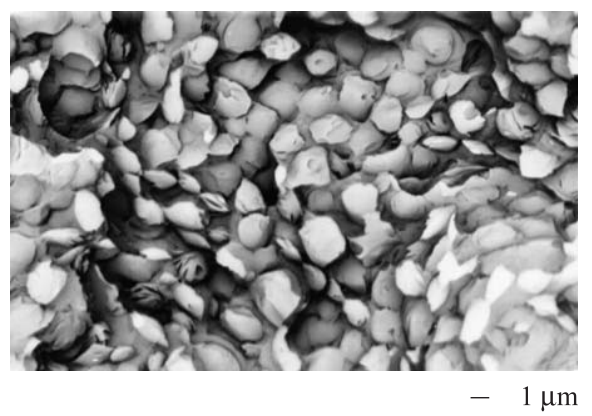

(d)

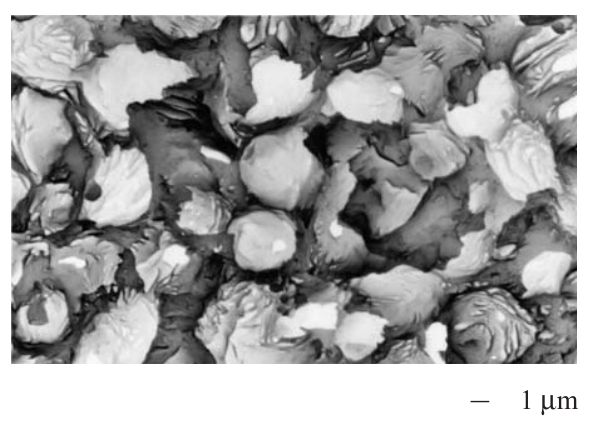

Fig. 4. The SEM micrographs of microporous films formed by $\mathrm{PCL}_{60} \% \mathrm{PEG}_{40}$ plasticized by various amount of castor oil followed by leaching in an aqueous medium: (a) $0 \%$, (b) $5 \%$, (c) $10 \%$, and (d) $20 \%$. 
interaction between PCL and PEG. Na et al. [19] have found the similar result, and they reported that poly $(\mathrm{L}-$ lactide) is immiscible with PCL but miscible with PEG. This conclusion indirectly implied that PCL is not miscible with PEG. Because PCL and PEG were immiscible, when mixing of both, PEG spontaneously dispersed in PCL as spherical droplets in order to diminish the surface energy between PCL and PEG interface $[20,21]$. The presence of castor oil would enhance this phenomenon in terms of enlargement of pore size when PEG is leaching out. Because the pore size of coating film played an important role on drug release from microporous-controlled tablets, the enlargement of pore size in microporous films provided positive effect on it.

\subsection{In vitro release study of plasticized microporous- controlled tablet}

The coated tablets were prepared by dip-coating method, and the feasibility of this method was evaluated by the weight change of tablets before and after coating. The change of weights of tablets after coating of castor oil-plasticized $\mathrm{PCL}_{60} / \mathrm{PEG}_{40 \%}$ blends was in the range of $3.44 \pm 0.03-3.60 \pm 0.03 \%$. The coefficients of variance of these values were less than $2.5 \%$. This result indicated that the current dipcoating method could achieve homogeneous coating of theophylline core tablets.

Fig. 5 shows the release of drug from tablets coated with unplasticized and plasticized $\mathrm{PCL}_{60} \% \mathrm{PEG}_{40 \%}$

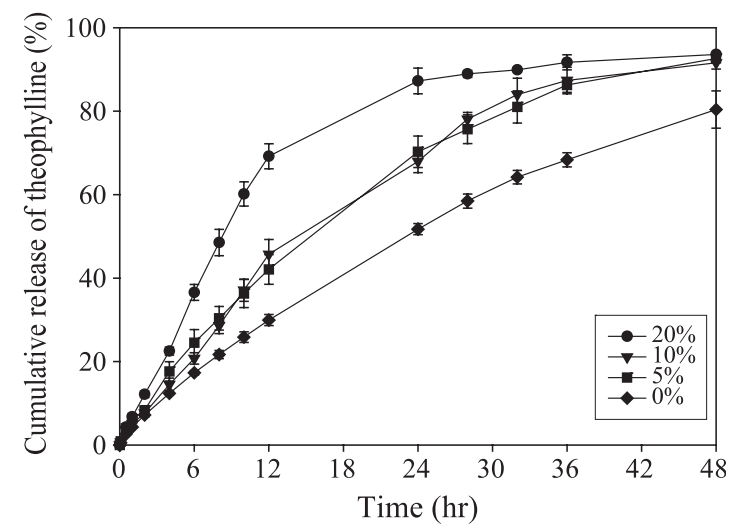

Fig. 5. The release of drug from microporous-controlled tablets coated by $\mathrm{PCL}_{60} / \mathrm{PEG}_{40 \%}$ blends plasticized by $0 \%(\diamond), 5 \%$ ( $)$, $10 \%(\boldsymbol{\nabla})$, and $20 \%(\bullet)$ of castor oil.
Table 1

The $k$ and $n$ values related to theophylline released from plasticized microporous-controlled tablets

\begin{tabular}{llll}
\hline $\begin{array}{l}\text { Castor oil } \\
(\% \mathrm{w} / \mathrm{w})\end{array}$ & $\begin{array}{l}k \\
\left(\% \mathrm{~h}^{-n}\right)\end{array}$ & $n$ & $\mathrm{r}^{2}$ \\
\hline 0 & 4.125 & 0.796 & 0.999 \\
5 & 6.092 & 0.766 & 0.996 \\
10 & 5.275 & 0.808 & 0.994 \\
20 & 6.938 & 0.921 & 0.997 \\
\hline
\end{tabular}

blends. The percentage of drug released was increased dependent of the amount of plasticizer incorporated, and the fastest release of drug was observed from tablets coated by $20 \%$ castor oil-plasticized $\mathrm{PCL}_{60 \%} /$ $\mathrm{PEG}_{40 \%}$ blend. The release of drug up to $70 \%$ was further fitted by Eq. (1); and the release rate constants and related $n$ values of drug from microporouscontrolled tablets plasticized by $0-20 \%$ of castor oil are listed in Table 1. The release rate constant of drug increased in the presence of plasticizer especially at $20 \%$ level and the drug release mechanism were switched from anomalous diffusion to near-constant release when $20 \%$ of castor oil was added. Because the release of drug from microporous-controlled tablets was mainly controlled by the pore size and the total number of pores created by PEG leaching out, the enlargement of pore size of microporous films by castor oil played an important role to account for this release outcome. On the other hand, the partition coefficient of theophylline from PCL film was determined to be 0.095 . In other words, about $10 \%$ of theophylline could partition into PCL and directly diffuse through PCL domain rather than from micropores. This is to say, the change of thermal and mechanical properties of films due to plasticizer effect influenced the release of drug from micropores and the diffusion through PCL both pathways in terms of enhancing drug release from the plasticized microporous-controlled tablets.

\section{Conclusion}

The influence of plasticizer on the release of theophylline from microporous-controlled tablets was demonstrated. The fatty plasticizer, castor oil, altered the thermal and mechanical performance and pore size of microporous films via soluble in PCL domain, which resulted in the release of theophylline from 
plasticized-coated tablets, which in turn enhanced and closed to a constant release pattern.

\section{Acknowledgments}

This work was supported by National Science Council in Taiwan (NSC 92-2320-B-002-146).

\section{References}

[1] R.E. Kesting, Polymer Solutions, Synthetic Polymeric Memtablet Branes, A Structural Perspective, Wiley, New York, 1985.

[2] G. Santus, R.W. Baker, Osmotic drug delivery: a review of the patent literature, J. Control. Release 35 (1995) 1-21.

[3] L.A. Felton, J.W. McGinity, Adhesion of polymeric films to pharmaceutical solids, Eur. J. Pharm. Biopharm. 47 (1999) $3-14$.

[4] S.Y. Lin, K.S. Chen, L. Run-Chu, Organic esters of plasticizers affecting the water absorption, adhesive property, glass transition temperature and plasticizer permanence of eudragit acrylic films, J. Control. Release 68 (2000) 343-350.

[5] A. Flosser, K. Kolter, H.B. Reich, G. Schepky, Variation of composition of an enteric formulation based on Kollicoat MAE 30 D, Drug Devel. Ind. Pharm. 26 (2000) 177-187.

[6] T.U. Okarter, K. Singla, The effects of plasticizers on the release of metoprolol tartrate from granules coated with a polymethacrylate film, Drug Devel. Ind. Pharm. 26 (2000) $323-329$.

[7] M. Anker, M. Stading, A.M. Hermansson, Effects of $\mathrm{pH}$ and the gel state on the mechanical properties, moisture contents, and glass transition temperatures of whey protein films, J. Agric. Food Chem. 47 (1999) 1878-1886.

[8] L.E. Appel, J.H. Clair, G.M. Zentner, Formulation and optimization of a modified microporous cellulose acetate latex coating for osmotic pumps, Pharm. Res. 9 (1992) 1664-1667.

[9] N. Sarisuta, R. Saowakontha, C. Ruangsuksriwong, Effects of surfactant on release characteristics of clonidine hydrochloride from ethylcellulose film, Drug Devel. Ind. Pharm. 25 (1999) $373-377$.
[10] M.A. Repka, J.W. McGinity, Influence of vitamin E TPGS on the properties of hydrophilic films produced by hot-melt extrusion, Int. J. Pharm. 202 (2000) 63-70.

[11] J. Hutchings, D.E. Sakr, Influence of $\mathrm{pH}$ and plasticizers on drug release from ethylcellulose pseudolatex coated pellets, J. Pharm. Sci. 83 (1994) 1386-1390.

[12] J. Siepmann, F. Lecomte, R. Bodmeier, Diffusion-controlled drug delivery systems: calculation of the required composition to achieve desired release profiles, J. Control. Release 60 (1999) 379-389.

[13] M. Wesseling, R. Bodmeier, Influence of plasticization time, curing conditions, storage time, and core properties on the drug release from Aquacoat-coated pellets, Pharm. Dev. Technol. 6 (2001) 325-331.

[14] E. Fukui, N. Miyamura, T. Yoneyama, M. Kobayashi, Drug release from and mechanical properties of press-coated tablets with hydroxypropylmethylcellulose acetate succinate and plasticizers in the outer shell, Int. J. Pharm. 217 (2001) 33-43.

[15] B.S. Rao, K.V. Murthy, Studies on rifampicin release from ethylcellulose coated nonpareil beads, Int. J. Pharm. 231 (2002) 97-106.

[16] M. Tarvainen, R. Sutinen, S. Peltonen, P. Tiihonen, P. Paronen, Starch acetate- a novel film-forming polymer for pharmaceutical coatings, J. Pharm. Sci. 91 (2002) 282-289.

[17] W.J. Lin, H.K. Lee, Design of a microporous controlled delivery system for theophylline tablets, J. Control. Release 89 (2003) 179-187.

[18] R.W. Korsmeyer, R. Gurny, E. Doelker, P. Buri, N.A. Peppas, Mechanisms of solute release from porous hydrophilic polymers, Int. J. Pharm. 15 (1983) 25-35.

[19] Y.H. Na, Y. He, X. Shuai, Y. Kikkawa, Y. Doi, Y. Inoue, Compatibilization effect of poly( $\varepsilon$-caprolactone)- $b$-poly(ethylene glycol) block copolymers and phase morphology analysis in immiscible poly(lactide)/poly( $\varepsilon$-caprolactone) blends, Biomacromolecules 3 (2002) 1179-1186.

[20] W.J. Lin, D.R. Flanagan, R.J. Linhardt, A novel fabrication of poly( $\varepsilon$-caprolactone $)$ microspheres from blends of poly $(\varepsilon$ caprolactone) and polyethylene glycols, Polymer 40 (1999) 1731-1735.

[21] W.J. Lin, C.H. Lu, Characterization and permeation of microporous poly( $\varepsilon$-caprolactone) films, J. Membr. Sci. 198 (2002) 109-118. 